Received: 28 July 2015

Accepted: 12 August 2015

Published online: 11 September 2015

(C) Springer-Verlag Berlin Heidelberg and ESICM 2015

F. Schortgen $(\varpi)$

Service de réanimation médicale, CHU Henri Mondor-APHP, 51 avenue du Maréchal de Lattre de Tassigny, 94000 Créteil, France

e-mail: frederique.schortgen@hmn.aphp.fr

Long-term mortality and sequelae have become a leading issue in the growing number of ICU survivors. Renal outcome after an episode of acute kidney injury (AKI) illustrates the significant risk of non-recovery of organ function after discharge. AKI and chronic kidney disease (CKD) are now recognized as an interconnected syndrome. Epidemiologic evidence shows that AKI is an independent risk for the occurrence or the progression of $\mathrm{CKD}$, and joint physiopathological mechanisms have been described [1]. Today, studies on AKI recovery suffer from the same issue as research on AKI several years ago, when a consensual definition was lacking. The use of different definitions of AKI recovery (Table 1) precludes any synthetic analysis of the literature and a wide range of post-AKI non-recovery has been reported, from 0.03 to 72 cases per 100 person-years [2-4]. This heterogeneity not only depends on the definition used but also on case-mix. Important risk factors of AKI non-recovery have been identified such as pre-existing CKD, age and AKI severity $[2,3,5]$.
It is intuitive to define recovery by the disappearance of a disease. Because AKI stages are defined by a percentage of serum creatinine (Screat) increase, such a simple definition would not correspond to complete recovery. A patient with a baseline Screat of $1.5 \mathrm{mg} / \mathrm{dL}$ and a maximal Screat of $2.5 \mathrm{mg} / \mathrm{dL}$ (AKI stage 1 [6]) would be considered as reaching renal recovery if his/her Screat return below $2.25 \mathrm{mg} / \mathrm{dL}$. Defining partial and complete recovery appears, therefore, necessary. In the most severe cases, recovery of a sufficient renal function allowing renal replacement therapy (RRT) independency is also a pertinent outcome that should be included.

In a recent issue of Intensive Care Medicine, Schetz and collaborators provide useful information on the impact of the criteria used to define AKI recovery [7]. In the subgroup of 1310 patients randomized in the "early versus late parenteral nutrition in critically ill adults" study [8] who developed AKI, they compared AKI recovery rate at hospital discharge, according to different

Table 1 Common criteria used to define renal recovery

\section{Renal function}

Discontinuation of dialysis

eGFR $>60 \mathrm{~mL} / \mathrm{min}$

Disappearance of AKI criteria

Return to \pm 10 or $20 \%$ of baseline Screat/eGFR

Return to baseline Screat/eGFR

Time point

ICU discharge

Hospital discharge

30 days

3 months

$>3$ months

Population

Patients with AKI

Patients with AKI requiring RRT

Survivors from AKI 
definitions. The severity of the creatinine-based definition strongly affected the complete recovery rate. The least severe tested definition was the disappearance of AKI criteria [6] and yield for $79 \%$ of recovery. When using the most stringent threshold corresponding to the return of Screat to baseline, recovery rate was only $42 \%$. Baseline Screat is, however, frequently unknown [2]. The authors compared recovery rate in patients in whom pre-existing renal function was available using either the true or the calculated Screat value obtained from the Modification of Diet in Renal Disease formula [6]. A large difference was found in the recovery rate which was significantly lower when using the calculated Screat. This difference was mainly related to the patients with pre-existing CKD in whom the calculated Screat underestimated baseline Screat. In the absence of known baseline renal function, it is impossible to differentiate de novo CKD and pre-existing unknown $\mathrm{CKD}$. In any case, de novo CKD diagnosis or the detection of unknown pre-existing CKD will require similar management with a referral to nephrologists when needed.

A definition of AKI recovery based on Screat evolution is hampered by the decrease in the creatinine production rate during an ICU stay $[9,10]$. Schetz and collaborators observed that, at hospital discharge, $40 \%$ of the patients had a Screat lower than baseline and $20 \%$ of CKD patients had a paradoxical normalization of their renal function. Many factors affect muscle wasting in critical illness and, therefore, can influence Screat. This is particularly true for older patients in whom a definition based on Screat overestimates the recovery rate [3]. In the Schetz study, a similar overestimation was found in CKD patients in whom recovery was paradoxically higher than in patients without CKD, except when RRT independency was considered in the definition. A higher muscle wasting in CKD patients might explain this observation. Another important factor affecting muscle wasting is the length of stay (LOS). Unsurprisingly, patients "healed" from their CKD at hospital discharge had a longer LOS. For all these reasons, a definition of short-term AKI recovery based on Screat will always overestimate the glomerular filtration rate. In critically ill patients, complete AKI recovery should result in a post-AKI Screat lower than baseline. A stringent threshold for the decrease in Screat after an AKI episode should therefore be chosen. As for defining AKI, it appears uncertain that the use of biomarkers could overcome the limitations of Screat [11].

The renal recovery definition should include a standardized time point (Table 1). While a short period of time seems sufficient to become independent from RRT [12], different patterns of glomerular filtration rate evolution have been described after AKI and its stabilization may require up to 1 year [5]. While not studied by Schetz and collaborators, it is likely that the time point for renal function assessment influences the recovery rate for two main reasons. First, the longer the delay, the higher the odds of recovery. A systematic review found that AKI recovery was $67 \%$ at hospital discharge and $76 \%$ when a delayed time point was used [3]. The odds of late recovery may, however, depend on the baseline renal reserve [3]. Conversely, delayed assessment may increase the probability of new renal insults and post-discharge AKI episodes that could decrease the odds of recovery [13]. Second, a longer LOS will result in a more pronounced artificial decrease in Screat. While it seems preferable to define a fixed time point, assessing renal recovery "at least" at hospital discharge may correspond to a pragmatic time point at which patients with non- or partial recovery can be detected.

Patient evolution after AKI corresponds to a multistate model that must be considered. Death, dialysis dependency, partial and complete recovery are all possible outcomes. Schetz and collaborators addressed the question of studying recovery in survivors only. This point appears crucial because the absence of recovery was twofold higher in non-survivors. To determine the volume of patients who will require a nephrological follow-up, AKI recovery should be assessed in survivors only. When testing interventions, death that precludes new or worsening CKD must be considered as a competing risk, and specific statistical methodologies are required to avoid wrong conclusions [14]. For instance, in some studies showing a better renal outcome with continuous RRT, the significant difference was merely explained by a higher mortality in patients managed with continuous rather than intermittent RRT. When using a composite end point (death and/or renal recovery), the difference was no longer significant [15].

Unfortunately, a validation of the definitions tested by Schetz and collaborators is lacking. Such validation could be based on the risk of long-term death or end stage renal disease (ESRD) [16]. Pannu and collaborators tested different thresholds of recovery from within $5 \%$ of baseline Screat to $>55 \%$, and found that the adjusted risk was significant for a threshold $>55 \%$ for death and $>25 \%$ for ESRD [16].

Schetz and collaborators clearly demonstrated that the use of different definitions leads for strong differences in the epidemiology of AKI recovery. Although a definition 
is never perfect, it is urgently needed. This definition should facilitate the estimate of the exact burden of postAKI non-recovery and the assessment of preventive measures of long-term renal sequelae. In clinical practice, such a definition should help to detect patients with persistent renal dysfunction who could benefit from referral to nephrologists $[17,18]$.

\section{Compliance with ethical standards}

Conflicts of interest None.

\section{References}

1. Chawla LS, Eggers PW, Star RA, Kimmel PL (2014) Acute kidney injury and chronic kidney disease as interconnected syndromes. N Engl J Med 371:58-66

2. Sawhney S, Mitchell M, Marks A, Fluck N, Black C (2015) Long-term prognosis after acute kidney injury (AKI): what is the role of baseline kidney function and recovery? A systematic review. BMJ Open 5:e06497

3. Schmitt R, Coca S, Kanbay M, Tinetti ME, Cantley LG, Parikh CR (2008) Recovery of kidney function after acute kidney injury in the elderly: a systematic review and meta-analysis. Am J Kidney Dis 52:262-271

4. Coca SG, Singanamala S, Parikh CR (2012) Chronic kidney disease after acute kidney injury: a systematic review and meta-analysis. Kidney Int 81:442-448

5. Chawla LS, Amdur RL, Amodeo S, Kimmel PL, Palant CE (2011) The severity of acute kidney injury predicts progression to chronic kidney disease. Kidney Int 79:1361-1369

6. Kellum JA, Lameire N (2013) Diagnosis, evaluation, and management of acute kidney injury: a KDIGO summary (Part 1). Crit Care 17:204

7. Schetz M, Gunst J, De Vlieger G, Van den Berghe G (2015) Recovery from AKI in the critically ill: potential confounders in the evaluation. Intensive Care Med 41:1648-1657. doi: 10.1007/s00134-015-3946-3
8. Casaer MP, Mesotten D, Hermans G, Wouters PJ, Schetz M, Meyfroidt G, Van Cromphaut S, Ingels $C$,

Meersseman P, Muller J, Vlasselaers D, Debaveye Y, Desmet L, Dubois J, Van Assche A, Vanderheyden S, Wilmer A, Van den Berghe G (2011) Early versus late parenteral nutrition in critically ill adults. N Engl J Med 365:506-517

9. Schetz M, Gunst J, Van den Berghe G (2014) The impact of using estimated GFR versus creatinine clearance on the evaluation of recovery from acute kidney injury in the ICU. Intensive Care Med 40:1709-1717

10. Prowle JR, Kolic I, Purdell-Lewis J, Taylor R, Pearse RM, Kirwan CJ (2014) Serum creatinine changes associated with critical illness and detection of persistent renal dysfunction after AKI. Clin J Am Soc Nephrol 9:1015-1023

11. Srisawat N, Wen X, Lee M, Kong L, Elder M, Carter M, Unruh M, Finkel K, Vijayan A, Ramkumar M, Paganini E, Singbartl K, Palevsky PM, Kellum JA (2011) Urinary biomarkers and renal recovery in critically ill patients with renal support. Clin J Am Soc Nephrol 6:1815-1823

12. Lin YF, Ko WJ, Chu TS, Chen YS, Wu VC, Chen YM, Wu MS, Chen YW, Tsai CW, Shiao CC, Li WY, Hu FC, Tsai PR, Tsai TJ, Wu KD (2009) The 90-day mortality and the subsequent renal recovery in critically ill surgical patients requiring acute renal replacement therapy. Am J Surg 198:325-332
13. Thakar CV, Christianson A, Himmelfarb J, Leonard AC (2011) Acute kidney injury episodes and chronic kidney disease risk in diabetes mellitus. Clin J Am Soc Nephrol 6:2567-2572

14. Koller MT, Raatz H, Steyerberg EW, Wolbers M (2012) Competing risks and the clinical community: irrelevance or ignorance? Stat Med 31:1089-1097

15. Bell M, Granath F, Schon S, Ekbom A, Martling CR (2007) Continuous renal replacement therapy is associated with less chronic renal failure than intermittent haemodialysis after acute renal failure. Intensive Care Med 33:773-780 (Epub 2007 Mar 2016)

16. Pannu N, James M, Hemmelgarn B, Klarenbach S, Alberta Kidney Disease N (2013) Association between AKI, recovery of renal function, and longterm outcomes after hospital discharge. Clin J Am Soc Nephrol 8:194-202

17. Harel Z, Wald R, Bargman JM, Mamdani M, Etchells E, Garg AX, Ray JG, Luo J, Li P, Quinn RR, Forster A, Perl J, Bell CM (2013) Nephrologist follow-up improves all-cause mortality of severe acute kidney injury survivors. Kidney Int 83:901-908

18. Goldstein SL, Jaber BL, Faubel S, Chawla LS (2013) AKI transition of care: a potential opportunity to detect and prevent CKD. Clin J Am Soc Nephrol 8:476-483 